\title{
Familiar shapes attract attention in figure-ground displays
}

\author{
Rolf A. Nelson \\ Wheaton College, Norton, Massachusetts \\ AND \\ STEPHEN E. PALMer \\ University of California, Berkeley, California
}

\begin{abstract}
We report five experiments that explore the effect of figure-ground factors on attention. We hypothesized that figural cues, such as familiar shape, would draw attention to the figural side in an attentional cuing task using bipartite figure-ground displays. The first two experiments used faces in profile as the familiar shape and found a perceptual advantage for targets presented on the meaningful side of the central contour in detection speed (Experiment 1) and discrimination accuracy (Experiment 2). The third experiment demonstrated the figural advantage in response time (RT) with nine other familiar shapes (including a sea horse, a guitar, a fir tree, etc.), but only when targets appeared in close proximity to the contour. A fourth experiment obtained a figural advantage in a discrimination task with the larger set of familiar shapes. The final experiment ruled out eye movements as a possible confounding factor by replicating the RT advantage for targets on the figural side of face displays when all trials containing eye movements were eliminated. The results are discussed in terms of ecological influences on attention, and are cast within the framework of Yantis and Jonides's (1984) hypothesis that attention is exogenously drawn to the onset of new perceptual objects. We argue that the figural side constitutes an "object," whereas the ground side does not, and that figural cues such as shape familiarity are effective in determining which areas represent objects.
\end{abstract}

The phenomenon of figure-ground organization in visual perception was first demonstrated and investigated in a classic monograph by Rubin (1915/1958). He began with the observation that simple displays containing two adjacent regions separated by a single luminance edge produce a strong tendency for observers to perceive one region as "figure" and the other as "ground." Exactly what makes up the processes that underlie figure-ground organization remains elusive, however, because there are actually several perceptual components that accompany this phenomenal organization. One is shape: The figure is shaped by the contour, whereas the ground is not. Measurable consequences of this "border ownership" aspect of figure-ground organization include better memory for the shape of the figure than for the shape of the ground (Davis, 1985; Rubin, 1915/1958), and faster and more accurate shape judgments about the figure region than about the ground region (Baylis \& Driver, 1995). A second aspect of figure-ground organization involves perceived depth across the edge: The figure appears closer than the ground. Some researchers have taken relative depth at the contour to be the primary, defining component of figureground organization, especially in discussions of the physiological basis of figure-ground organization (e.g., Hupé et al., 1998; Lamme, 1995; von der Heydt, Zhou, \&
Friedman, 2000). A third aspect of figure-ground organization is a weak form of amodal completion: The ground is at least partly completed behind the figure, even though there is no direct sensory evidence of this unseen portion. Whereas the area of the figure ends at the contour, the area of the ground continues behind that contour and appears to be filled in by an extrapolation process.

Yet another aspect of figure-ground organization, perhaps less well understood than the three just mentioned, is the role of attention. Although Rubin (1915/1958) did not mention attention explicitly, he did state that the figure seems to pervade consciousness more than the ground, which is consistent with some sort of attentional involvement. More recently, several textbooks have alluded more directly to a relation between attention and figure-ground organization. For example, Matlin (1983) states that the figure is more dominant and brighter, aspects that are often attributed to attended regions or objects. ${ }^{1}$ Coren, Ward, and Enns (1999) describe figures as being "richer" than the ground and as being processed in more detail. Sternberg (1996) states that a figure is any object that is "highlighted" against an "unhighlighted" background, and that it is possible, but difficult, to attend to the ground. Indeed, many of the established differences between figure and ground are similar to those between attended and

R.A.Nelson, rnelson@wheatonma.edu 
unattended regions: The figure is remembered better, is processed more quickly, is more clearly defined, pervades consciousness, and so forth. This leads naturally to the question: What exactly is the relation between figureground organization and attention?

One claim regarding this relationship is that attention, like surroundedness, symmetry, and convexity, is a factor that influences figural status. ${ }^{2}$ The claim is that attending to an otherwise ambiguous region makes it more likely to be seen as figure. Baylis and Driver (1995) tested this conjecture by measuring performance on an edge-matching task when attention was directed to one side or the other, either by presenting an arrow pointing to the cued side (the endogenous cuing condition) or by flashing a small bright cue just beyond the region where one side of the figure-ground display was to appear (the exogenous cuing condition). They found a figural advantage in their shapematching task for an endogenously cued side, but no such advantage for an exogenously cued region. A replication and extension by Vecera, Flevaris, and Filapek (2004) did find an advantage for the exogenously cued side, however, provided that the cue was presented inside the region in which the figure-ground display was to appear rather than just near it. At least in ambiguous displays, then, attention can be viewed as a factor that influences figure-ground assignment: The attended side is biased toward being seen as figure. Neuropsychological studies on patients with unilateral neglect reinforce this claim. Peterson, Gerhardstein, Mennemeier, and Rapcsak (1998) found that these patients, who experience difficulty attending to one side of space, are less likely to perceive a region on their neglected side as figure.

A second possible relation between figure-ground organization and attention, and the concern of the present studies, is that classical figure-ground cues may draw attention to the figural side of the contour, thereby producing more rapid and accurate processing of targets on the figural side. Previous research on the question of figureground influences on target detection has produced somewhat confusing and counterintuitive results, however. In a series of often cited experiments, Gelb and Granit (1923) reported higher thresholds for detecting a low-contrast target on the figure than for one on the ground. It is worth examining Gelb and Granit's experiment in detail, because the results have frequently been misrepresented as a "figural inferiority" effect (e.g., Koffka, 1935; Osgood, 1953; Wong \& Weisstein, 1982). First, the stimulus they used (a fan with four equal-sized "blades" on a larger surround) included a geometrical confound: Each blade of the figural region was surrounded on all three sides by luminance contours, whereas each segment of the ground region between the blades was only surrounded on two sides by such contours. If nearby luminance contours tend to mask perception of the low-contrast target, detection should have been better in their ground condition than in their figure condition simply due to differential masking effects (Craik \& Zangwill, 1939). Second, the participants (of which there were only 4) were shown some blocks of trials in which the low-contrast test light always appeared in the figural region and other blocks of trials in which it always appeared in the ground region. Therefore, the participants may have rapidly learned the location in which the spot of light was to appear and focused their attention on that area, making an attentional interpretation of the results unlikely.

Later work by Wong and Weisstein (1982) provided evidence suggesting that, contrary to Gelb and Granit's (1923) claim, perception in the figural region has an advantage over perception in the ground region. In their experiment, an ambiguous face-vase stimulus with line (rather than luminance) contours was used. The stimulus could either be perceived as a vase or as two opposing faces in profile, depending on the figural assignment of the two contours. Observers performed better on a line-discrimination task when the line segment was in a region that they were currently perceiving as figure than when it was in a region they were currently perceiving as ground. This could not be explained by lower-level sensory factors (as in the Gelb \& Granit, 1923, studies) because the stimulus was exactly the same in both conditions; the only difference was the side perceived as figure.

Wong and Weisstein (1982) interpreted their results as indicating that there is a perceptual advantage for processing information in a figural region. They did not appear to consider whether attention might have been responsible for the differences they reported (although Peterson \& Gibson, 1993, did). It remains entirely possible that, when participants perceived the vase as figure, for example, attention was drawn to the vase and away from the faces, and that this shift in attention actually caused the perceptual advantage they reported. This could have happened in either of two ways. First, attention might have preceded figural assignment. That is, a region might have been the first attended to, similar to the effects of exogenous cues discussed above, causing it then to be perceived as figure. As a side effect of this attention, performance was enhanced in the region. Second, figural assignment might have occurred first, and attention might then have been drawn to the figural region, causing the perceptual advantage. These two possibilities are not mutually exclusive. It certainly might be the case that, in an otherwise ambiguous display, attention can cause a given region to be perceived as figure, and figural assignment can also draw attention to the same region, in a mutually facilitatory feedback loop.

The purpose of the present set of experiments was to examine more directly how cues to figure-ground organization relate to the allocation of attention. Specifically, do the same cues that cause one side of a contour to be perceived as figure (meaningfulness, surroundedness, symmetry, etc.) also draw attention to that side? It is important to note that, whereas it may be the case that whatever is perceived as the figure is also attended, we do not claim that "attention is drawn to the figure." The figure-ground distinction is a purely phenomenological one, and in the interests of maintaining an objective measure of what draws attention, we only make claims about the effects of the manipulated figural cues. Nevertheless, in the interest of brevity, we will refer to one side as figure rather than "the region influenced by shape cues to be most often 
perceived as figure." As noted above, there are several suggestive results regarding this relation in the literature, but none of them are definitive. Preliminary evidence from neurophysiology may also suggest a link between attention and figure-ground organization. When Sugihara, Qiu, and von der Heydt (2004) recorded from cells in area V2 of monkey cortex that responded to border ownership, they found stronger attentional modulation on the preferred border ownership side than on the nonpreferred side. Our primary goal in the present experiments was to determine whether well-established figural cues, such as shape familiarity (see, e.g., Peterson \& Gibson, 1994), draw attention as measured by a well-established index of attention, such as reaction time (RT) in an attentional cuing paradigm (e.g., Jonides, 1981; Posner, Nissen, \& Ogden, 1978).

The methods we used to study whether figural cues influence attention are derived from the spatial cuing paradigms developed by Posner (e.g., Posner, 1978, 1980; Posner et al., 1978) and Jonides (e.g., 1980, 1981, 1983). In these experiments, the benefit of attending to a region in space is measured by faster responses to targets presented in the attended region than in the nonattended region. Posner and colleagues (e.g., Posner et al., 1978) found that certain endogenous cues, such as a central arrow pointing to a target location, reduced RT to detect a target when it appeared in the cued location, but only if the cue was predictive of the target's location. Jonides and colleagues (e.g., Jonides, 1981; Yantis \& Jonides, 1984) found that certain exogenous cues, such as the sudden onset of an object, also reduced RT to detect the target, but they appeared to draw attention automatically, even if they were not predictive of the target's location.

In the present experiments, an analogy is drawn between the attentional effect of so-called "exogenous" or "pull" cues (e.g., brightening a bounded region) and the effect of figural cues (e.g., meaningfulness, convexity, size, and surroundedness). If such figural cues "pull" attention to the figural side of the contour, then detection should be faster for a target presented on the figure than for one on the ground. The basic procedure common to all experiments reported here is that a figure-ground stimulus appears and, after a variable delay, a target is presented in either the figure or the ground region. If figural cues in the stimulus draw attention to the figural side, superior speed and accuracy in detecting a target should be found when the target is located within the figural region rather than when it is located within the ground region.

\section{EXPERIMENT 1 Speed of Target Detection}

One powerful cue to the assignment of figural status is meaningfulness. When other stimulus factors (such as size, color, symmetry, and orientation) are equated, a region that has the familiar shape of a known class of objects is more likely to be perceived as the figure (Peterson \& Gibson, 1994; Peterson, Harvey, \& Weidenbacher, 1991). Rubin (1915/1958) mentioned that there were effects of previous experience on the perception of a figure, such that a region perceived as a figure in a prior viewing would be more likely to be perceived as a figure again. These memory effects are related to those of meaningfulness, because in order to be meaningful, a region must either have been seen before or be perceived as similar to something that has been seen before.

There is some evidence that the meaning of an object can act to automatically orient attention. For example, Christie and Klein (1995) have found better motion discrimination for a meaningful word than for a nonword (presented simultaneously) when probed $100 \mathrm{msec}$ after onset. Other work in the visual search literature indicates that detection time for faces can depend on such factors as emotional content (e.g., Hansen \& Hansen, 1988) and racial characteristics of the participant and the target (e.g., Levin, 1996).

In order to maximize the chance that the stimulus would be familiar to participants in the experiment, we used faces in profile as the meaningful shapes in Experiment 1. Because humans are especially adept at distinguishing and identifying them (see Maurer, Le Grand, \& Mondloch, 2002, for a review), faces should be particularly effective in biasing contour assignment.

\section{Method}

Participants. The participants were 13 undergraduate students at the University of California, Berkeley, who received course credit as part of a requirement for a psychology course. All had normal or corrected-to-normal vision.

Equipment. All of the experiments except Experiment 4 were conducted on a Dell Dimension 8200 computer with a Dell M990 monitor. Experiment 4 was conducted on a Dell Precision 370 computer with a Dell LCD monitor. Stimuli were presented with the software program Presentation. Responses in all experiments except Experiment 4 were made by pressing mouse buttons. Responses in Experiment 4 were made by pressing buttons on a Cedrus RB- 834 button box.

Design. The stimuli were rectangles divided into two regions of equal area, one of which was always in the shape of a face in profile. The two colors used for the two regions were red and blue. Two different faces were used for the experiment, one in each block of trials. The stimuli were presented equally often upright, upside down, and left-right reversed.

The participants were given instructions on the experimental procedure and seated approximately $35 \mathrm{~cm}$ from the computer monitor (although no chinrest was used to stabilize head position). The participant's task in the experiment was to respond as quickly as possible when an " $\mathrm{X}$ " appeared on either side of the figure-ground display. The event structure of each trial is shown in Figure 1. After a fixation cross indicated where the participants were to keep their eyes fixated, a figure-ground display appeared, centered in the place where the fixation cross had been. The fixation cross was positioned so that it was along the line where the contour would later appear (that is, not within either of the regions). The figure-ground display always consisted of one red and one blue region. These regions were not equated for luminance; however, targets were equally likely to appear in a red or blue region in the figure or the ground. After a variable stimulus onset asynchrony (SOA) of $0,150,250$, or $500 \mathrm{msec}$, a neutral gray target in the shape of an " $\mathrm{X}$ " subtending approximately $0.2^{\circ}$ of visual angle appeared on the figure-ground display near the shaped contour. As shown in Figure 1, there were three distances from fixation at which the target could appear. These distances were at $3^{\circ}, 5^{\circ}$, and $9^{\circ}$ of visual angle from fixation. For each distance, there were four targets: one on the figural side and one on the ground side, both above and below fixation. The variability in the targets' locations produced spatial uncertainty in the task, thus encouraging participants to attend broadly to the display. 


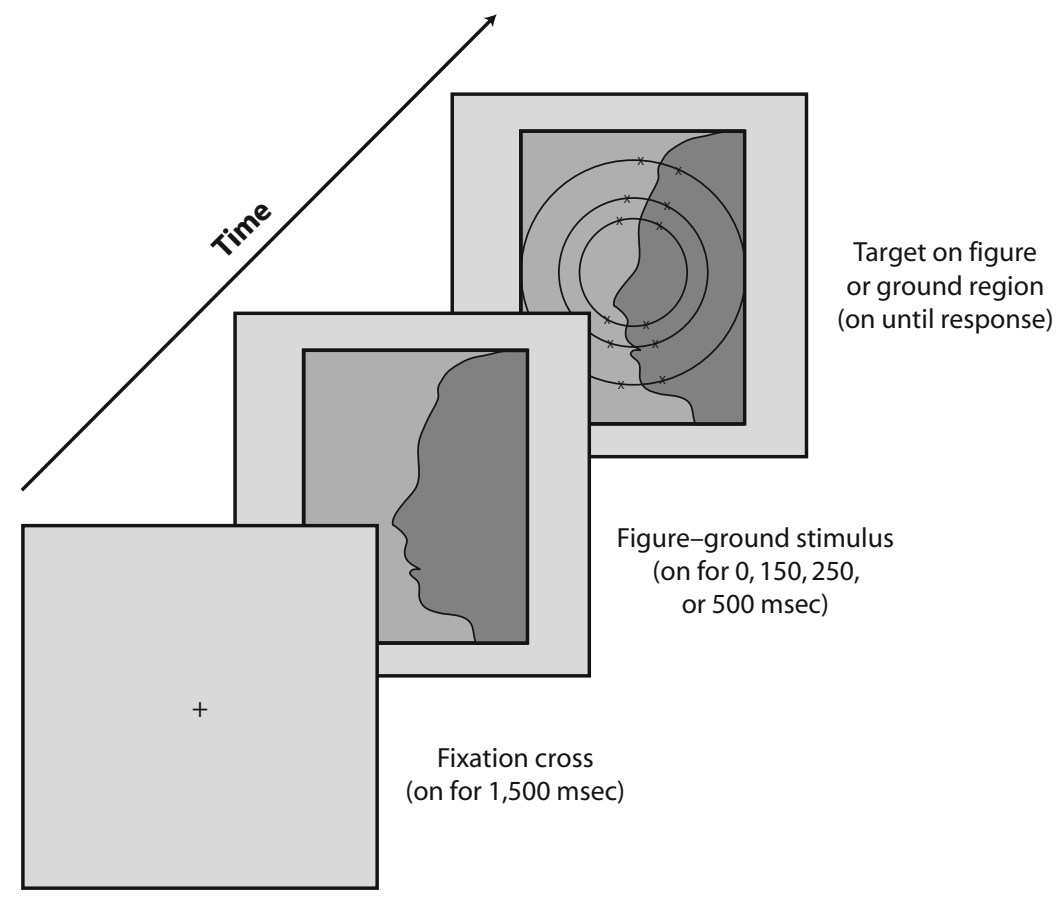

Figure 1. Sequence of events in Experiment 1. Target locations are indicated by the "X"s. Concentric circles, which were not present in the actual displays, are used to illustrate that corresponding targets on the figure and ground were placed equidistant from fixation.

Trials were presented in two blocks. Within each block, the same face contour was used, with two repetitions of all possible permutations of figure-ground condition (target on the figure/ground), orientation (upright/inverted), left-right reversal (left/right facing), color (red/blue), target locations (six locations, three above and three below fixation), and SOA (0/150/250/500 msec). These conditions were randomly presented within blocks.

After the experiment, the participants were shown a sheet of the 16 stimuli they had seen during the experiment, including both inverted and upright faces. After being briefly instructed about the nature of the distinction between figure and ground, they were asked which side they saw as figure. The purpose of this test was to determine the extent to which the participants perceived the meaningful sides as figures, both when they were upright and when they were inverted.

\section{Results and Discussion}

Figure 2 plots mean RTs for target detection in the figure and ground conditions over different SOAs. An ANOVA with the factors of figure-ground condition, SOA, target distance, and orientation was performed on the data. RTs to targets in the figural regions were significantly faster than those to targets in the ground regions $[F(1,12)=$ $26.25, p<.001]$. This difference was fairly constant over $\mathrm{SOA}$, as indicated by the lack of a reliable interaction between SOA and figure-ground condition $[F(3,36)<1]$. There was a main effect of SOA, such that participants were faster at detecting targets at longer SOAs $[F(3,36)=$ $32.68, p<.001]$. There was also an effect of target distance from fixation, such that responses were slower with increasing distance from fixation $[F(2,24)=12.06, p<$
.001], but this effect did not interact with figure-ground condition $[F(2,24)=1.52, p>.05]$.

There was no interaction between figure-ground condition and orientation $(F<1)$, suggesting that the familiarity of the facial profile drew attention even when it was inverted. The postexperimental test was consistent with this result, because every participant reported that they perceived every face region as figure, whether it was upright or inverted. This fact is perhaps not surprising, given that every display contained a face at some orientation and that the same displays were seen repeatedly. It does conflict, however, with prior results showing reduced figural perception for familiar shapes when they are presented upside down (e.g., Peterson \& Gibson, 1994). We will comment further on this issue in the General Discussion section after we have reported all of our results.

\section{EXPERIMENT 2 Accuracy of Target Discrimination}

Experiment 1 showed powerful effects of the figural factor of familiar shape on RT in a task similar to the well-known attentional cuing paradigm. This is consistent with the possibility that the visual system processes information from the figural region more quickly than information from the ground region. The next experiment was designed to determine whether the visual system also processes information from the figural region more accurately than information from the ground region. In Ex- 


\section{Experiment 1}

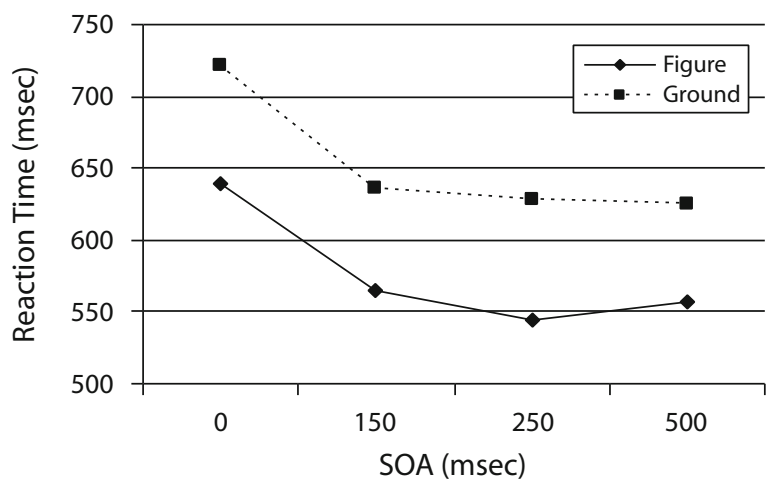

Figure 2. Results from Experiment 1: Participants were faster when the target appeared on the meaningful side of the contour (figure) than on the nonmeaningful side (ground).

periment 2, accuracy in discriminating between two possible targets (an " $\mathrm{X}$ " vs. a "Y") was measured for briefly presented, masked targets that appeared in figural regions versus ground regions.

Another change from Experiment 1 was that on some trials, after the participants had completed the discrimination task, they were asked to indicate which side they had perceived as figure. This additional task served the purpose of increasing the likelihood that the figure-ground status of the stimulus was relevant to the participants during the experiment.

\section{Method}

Participants. Fifteen undergraduate students at UC Berkeley participated in this experiment. They received course credit as part of a requirement for a psychology course. All of the participants had normal or corrected-to-normal vision.

Design. The stimuli were the same as those in Experiment 1. Again, one region was blue and the other region was red. There were two differently shaped faces, one in each block of trials. The stimuli were presented both upright and inverted.

The event structure of each trial was as follows. First, a fixation cross appeared on a gray screen for $500 \mathrm{msec}$. Next, a figure-ground display appeared on the screen, centered on the location of the previously presented fixation cross. Following a variable SOA $(0,150$, 250 , or $500 \mathrm{msec}$ ), a target appeared in either the meaningful or the nonmeaningful region. This target was either an "X" or a "Y," with equal probability. The target stayed on the screen for $80 \mathrm{msec}$ and was then replaced by a mask consisting of a pound sign (“\#”).

Targets were equally likely to appear in any of six locations for each region (meaningful or not). The targets were located in the same spatial locations as in Experiment 1. The participants responded by indicating whether they thought an "X" or a "Y" had been presented. They made their choice by pressing either the left ("X") or the right ("Y") mouse button with their right hand.

To encourage participants to perceive the displays as figures against a ground (that is, with the central contour shaping one region and not the other, and one region being perceived of as in front), they were told that they would be seeing pictures in which one side was a figure and the other side was ground, and they were shown a picture of an ambiguous display to illustrate how it might be possible to see either side as a figure. They were then told that on some trials, they would see a question mark instead of a fixation cross at the beginning. When this happened, they were to make a second response after they had indicated whether they had seen an "X" or a "Y." This second response was to indicate which side had looked like the figural region, and was made by pressing the mouse button (the same input device as for the first response) on the side that they remembered having perceived as the figure. After the experiment was completed, in order to verify that meaningfulness was an effective cue for figural status, we showed them all of the experimental displays on paper and asked them to indicate which side they perceived as figural.

Before beginning the experimental trials, the participants had 10 practice trials. When they responded that they understood the procedure, the experimental trials began.

There were two blocks of trials, each of which consisted of 384 trials $(2$ colors $\times 2$ up/down orientations $\times 2$ left/right orientations $\times 12$ target locations $\times 2$ repetitions). On $10 \%$ of these trials, the participants were asked to indicate their perception of figural status after the target discrimination response.

\section{Results and Discussion}

Figure 3 plots performance in the figure and ground regions with both the upright and inverted face stimuli in terms of average $d^{\prime}$, a measure of discriminability in signal detection theory (Green \& Swets, 1966). Discrimination was reliably better on the figure side than on the ground side $[F(1,14)=19.9, p<.001]$. As expected, responses were less accurate with increasing distance from fixation $[F(2,28)=30.42, p<.001]$, but this factor did not interact with figure-ground status $[F(2,28)=1.58$, $p>.05]$. However, a significant interaction did occur between orientation and meaningfulness $[F(1,14)=10.66$, $p=.006]$, such that the figural advantage was larger and statistically significant when the displays were upright $[t(14)=6.69, p<.0001]$, and smaller and not statistically significant when they were inverted $[t(14)=1.78$, $p>.05]$. This is the expected interaction between figureground condition and orientation, consistent with previous findings that the familiar, meaningful region is more likely to be seen as figure when the display is upright than when it is inverted (e.g., Peterson \& Gibson, 1994). Even so, it is surprising that this interaction appeared in the present experiment but not in Experiment 1. This result must be due either to differences in the task (i.e., detection in Experiment 1 vs. discrimination in Experiment 2)

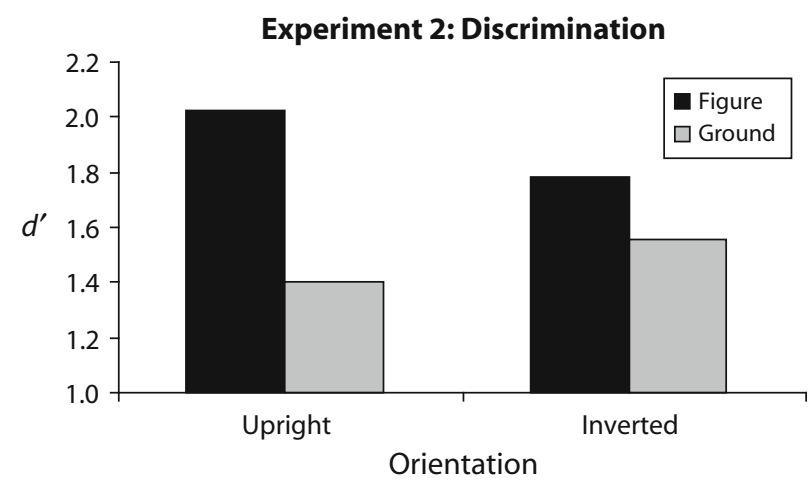

Figure 3. Results from Experiment 2: Performance on the meaningful region (figure) was better than on the nonmeaningful region (ground) in a discrimination task. Participants were also better at detection in the upright versus the inverted figure, but not the ground. 


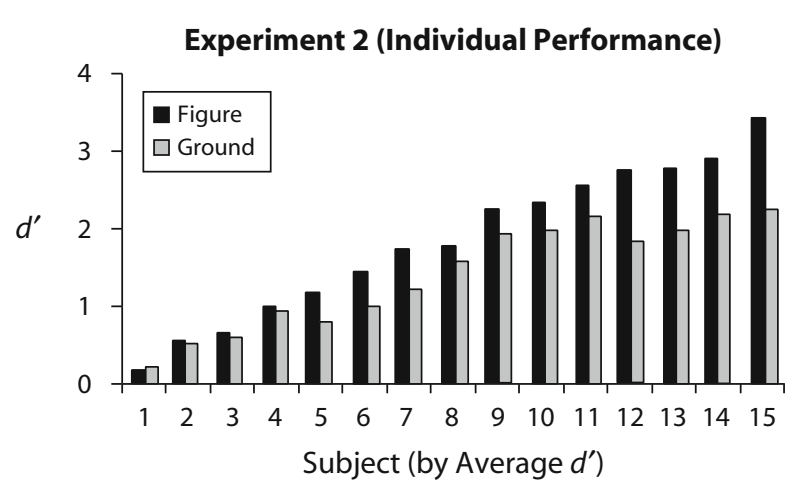

Figure 4. Individual performance in Experiment 2, plotted in ascending order of average $d$. Performance was better on the meaningful figure than on the nonmeaningful ground for participants who were able to make the discrimination above $d^{\prime}=1.0$.

or to the instruction in Experiment 2 to report the figural side on a subset of trials (which was not included in Experiment 1), because those were the only differences between the two experiments.

Figure 4 plots the $d^{\prime}$ results for individual participants in ascending order of average $d^{\prime}$. The only participant who failed to show better discrimination performance on the figural side responded essentially at random, and the magnitude of the figural advantage increased with overall accuracy across participants.

As in Experiment 1, every participant indicated that they saw the meaningful side as figure for all stimuli in a postexperimental verification, including both the upright and inverted faces.

\section{EXPERIMENT 3 Target Distance From the Contour}

In Experiments 1 and 2, all of the targets were located near the dividing contour. Previous pilot experiments had failed to show a figural advantage when the target was located near the center of the figural region, however. There were other differences between these experiments that made it difficult to determine whether distance from the contour per se was the important variable. We therefore designed a within-subjects study to determine how far the figural advantage spreads into the figure from the contour. Theoretically, there are reasons both to believe that it should extend throughout the entire region and to believe that it might be strongest near the contour and diminish toward the interior.

Classically, figural status is conceived as a property of regions: One region is designated as figure and the other as ground. Indeed, many of the well-known figural factorsincluding surroundedness, size, and symmetry - are properties of entire bounded regions rather than properties of local unbounded regions around edge segments. On this basis, one might reasonably expect the figural advantage to pervade the entire figural region. This logic is heavily based on the standard bipartite displays that dominate the figure-ground literature, however. In complex natural scenes, few objects are visible in their entirety against a farther background, because the boundaries of most visible objects are occluded along some portions and visible along others. This means that figure-ground organization in natural scenes is unlikely to be a truly regional property, but rather one that is localized near the edge itself. Such considerations lead to the alternate view that the figural advantage should occur primarily along the shared contour where the edge is assigned to that side. Consistent with this view, recent theories of figure-ground segregation posit a competition between figure and ground that occurs across the contour separating them (e.g., Peterson, 1999; Vecera \& O'Reilly, 1998), and it is possible that such competitive inhibition decreases with distance from the relevant contour(s). The purpose of the present experiment is therefore to determine the effect of target distance from the contour on speed of target detection.

Another change that was introduced in this experiment was the use of a wider set of familiar stimuli. To ensure that the effects found in Experiments 1 and 2 were not restricted to face-shaped contours, we used a more inclusive set of meaningful shapes. We chose 10 displays from the OMEFA set for which Peterson and her colleagues have derived an index of meaningfulness (Peterson, de Gelder, Rapcsak, Gerhardstein, \& Bachoud-Lévi, 2000; Peterson et al., 1998). One member of the set was a face in profile, but others included a sea horse, a wine glass, a fir tree, a guitar, and other familiar objects (for the complete set of OMEFA stimuli, the reader is referred to www.u.arizona .edu/ mapeters/).

\section{Method}

Participants. Nineteen undergraduate students at UC Berkeley participated in this experiment. They received course credit as part of a requirement for a psychology course. All had normal or corrected-to-normal vision.

Design. We selected 10 figure-ground stimuli from Peterson et al.'s (1998) OMEFA set, because they have already been proven to affect figure assignment and because their relative strengths in producing effects on figural assignment have already been measured by Peterson et al. (1998). Each shape could be either upright or inverted, each region could be either blue or red (the same colors as the stimuli in the previous experiments), and the meaningful region could be either on the left or on the right.

Targets were placed at varying distances from the dividing contour along the circumference of a virtual circle. For each stimulus, all of the targets were the same distance away from fixation, although the distance varied from stimulus to stimulus. There were six target locations presented below and six target locations presented above fixation. Among these, three targets were in the figure side and three targets in the ground side. These three targets were $0.6^{\circ}$, $1.2^{\circ}$, and $2.4^{\circ}$ of visual angle from the dividing contour.

SOAs were randomized between 0 and 1,000 $\mathrm{msec}$, in 100-msec intervals; otherwise, event structure was the same as in Experiment 1. The participants were again instructed to press the left mouse button as soon as they saw an "X" appear on the stimulus.

In order to ensure that the shapes were indeed perceived as meaningful, after the experiment, we showed the participants all of the experimental stimuli and asked two questions: "Which side did you see as figure?" and "What does that side look like, if anything?"

\section{Results and Discussion}

The percentage of participants who correctly named each stimulus in the postexperimental test correlated well with Peterson's (1998) “denotivity" index for individual items 
$(r=.84)$. Most of the participants correctly identified most of the stimuli, except for one with the shape of a wrench.

Figure 5 plots the results for figure versus ground conditions as a function of distance from the contour averaged over the 10 different displays. An ANOVA was conducted on the factors of SOA, figure-ground condition, orientation, and distance from the contour. Although there was a main effect of SOA, such that participants were faster at longer SOAs $[F(10,180)=3.6, p<.001]$, it did not interact significantly with any of the other variables, and will not be considered further.

The primary finding of interest was a significant interaction between figure-ground condition and distance from the contour $[F(2,36)=3.73, p<.05]$. The participants were faster at detecting the target on the figural side when it was at the two locations closer to the contour $\left[t(18)=1.8,2.0\right.$ for the distances of $0.6^{\circ}$ and $1.2^{\circ}$ of visual angle from the contour, respectively; both $p s<.05$ ], but not when it was at the farthest $\left(2.4^{\circ}\right)$ distance from the contour. The apparent reversal at the farthest distance was not significant $[t(18)<1]$.

In order to ensure that the effect was not driven entirely by the face stimulus, we did a further analysis with that stimulus removed. There was still an effect such that participants were faster when the target appeared in the meaningful region than when it appeared in the nonmeaningful region $[t(18)=2.29, p<.05]$. There also continued to be an interaction with distance, such that participants were faster in the figural region closer to the contour $[F(2,36)=$ $3.65, p<.05]$.

As in Experiment 1, but unlike in Experiment 2, no interaction between figure-ground condition and orientation was present $[F(1,18)<1]$. This fact indicates that the difference found between figure and ground did not change reliably when the stimuli were inverted.

\section{EXPERIMENT 4}

\section{Discrimination Using the OMEFA Stimulus Set}

One difficulty with interpreting the preceding results is in disentangling the effects of the stimulus set (faces

Experiment 3

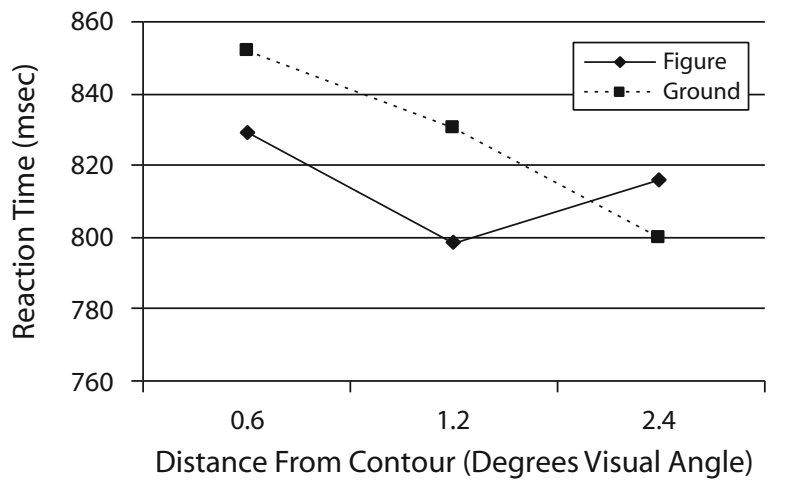

Figure 5. Results from Experiment 3: The advantage for the figural region was present only near the contour.

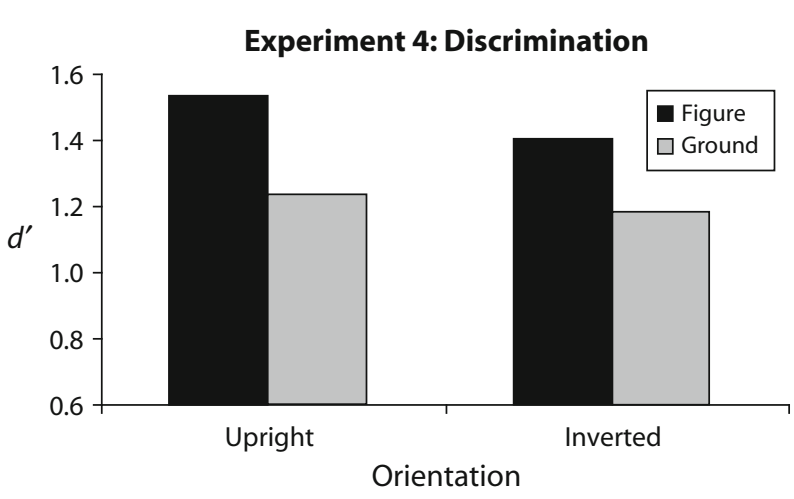

Figure 6. Results from Experiment 4: The discrimination task using the OMEFA stimuli.

or the OMEFA set), a probe to test the perception of figural status, and the measure of attention used (detection or discrimination). Therefore, we ran a discrimination experiment, with methodology similar to Experiment 2, using the larger stimulus set of Experiment 3. To ensure that the inclusion of a probe inquiring about figural status was not causing an endogenous strategy of attending to the figural side, we purposely did not include a probe in this experiment.

\section{Method}

Participants. Sixteen undergraduate students at Wheaton College, in Norton, Massachusetts, participated in this experiment in exchange for course credit for a psychology course. All had normal or corrected-to-normal vision.

Design. The set of meaningful stimuli was the same as in Experiment 3. There were four potential target locations for each stimulustwo on the meaningful side and two on the nonmeaningful side. Each target was $3.2^{\circ}$ of visual angle away from fixation and $0.7^{\circ}$ of visual angle away from the dividing contour.

The participants were secured in a chinrest and head stabilizer $30 \mathrm{~cm}$ away from the computer monitor. As in Experiment 3, SOAs were randomized between 0 and $1,000 \mathrm{msec}$, in 100 -msec intervals. The participants were instructed to press the left button if they saw an "X" or the right button if they saw a "Y" (both clearly labeled).

After 10 practice trials, there were 320 experimental trials, randomly presented $(2$ colors $\times 2$ orientations $\times 10$ stimuli $\times 4$ targets $\times 2$ target identities). There were no probe trials asking participants to identify which side they perceived as figure.

After the experiment, the participants were again shown the full set of upright stimuli and asked which side they perceived as figure and what the identity of the object on that side was.

\section{Results}

Again, the percentage of participants who correctly named each stimulus in the postexperimental test was highly correlated with Peterson's (1998) “denotivity" index $(r=.84)$. Of the 16 participants, 1 was excluded from analysis for a failure to perform the task according to instructions (repeatedly pressing buttons before the stimulus appeared on the screen). Three participants had overall discrimination at low levels $\left(d^{\prime}<.50\right)$ and were excluded from further analysis.

Figure 6 shows discrimination for the figural and ground sides when they were upright and inverted. Consistent with the previous findings, there was a main effect such that 


\section{Experiment 5}

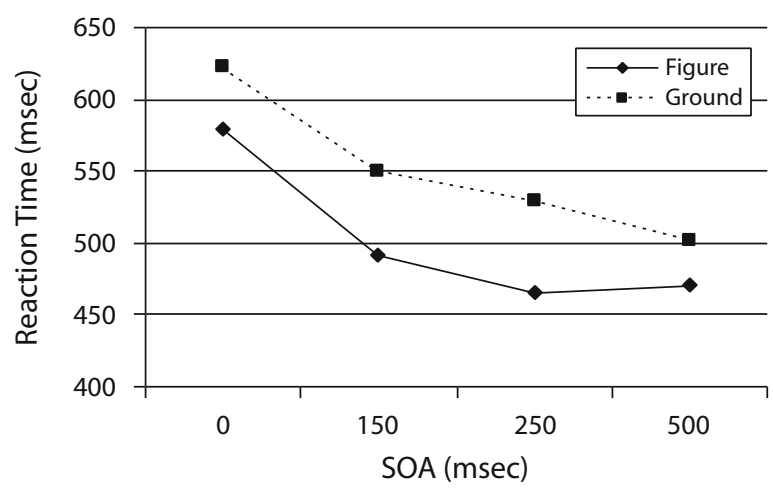

Figure 7. Results from Experiment 5: A replication of the first experiment, with eye movements controlled for.

participants were more accurate on the figural (meaningful) side (average $d^{\prime}=1.46$ ) than on the ground (nonmeaningful) side (average $\left.d^{\prime}=1.20\right)[F(1,11)=$ $6.68, p=.01]$. As in Experiment 2, the difference between discrimination accuracy on the figure and ground sides was slightly larger for the upright displays than for the inverted ones, but the interaction of orientation and figural status was not statistically reliable in the present data $[F(1,11)=.77, p>.05]$.

\section{EXPERIMENT 5 Eye Movements}

In all of the experiments reported thus far, the participants were instructed to keep their eyes fixated on the cross that appeared at the beginning of each trial, but no precautions were taken to eliminate eye movements, if they were actually made. No matter what instructions are given, participants might be unaware of their eye position and/or unable to stop their eyes from moving toward the figural region. If they actually moved their eyes toward the figural side, it would not be surprising that they were faster at detecting and/or more accurate at discriminating the target when it appeared on that side. Experiment 5 was undertaken to ensure that the figural effects observed were not due to eye movements. To do this, we directly monitored eye position with a video camera, and discarded trials in which eye movements occurred.

\footnotetext{
Method

Participants. Seven undergraduate students at UC Berkeley participated in this experiment. They received course credit as part of a requirement for a psychology course. All had normal or correctedto-normal vision.

Design. The design of the experiment was the same as that of Experiment 1, with respect to the stimuli (faces), task, target locations, and SOAs. The only differences were that the participants were positioned with their chins in a chinrest to stabilize their head position and that they were told that their eye movements were being monitored. The importance of keeping their eyes fixated on the fixation cross was also emphasized more strongly.

The eye-monitoring apparatus consisted of a small video camera mounted next to the experimental monitor. The video camera (ap-
}

proximately $45 \mathrm{~cm}$ from the participants' eyes) was connected to a separate black-and-white monitor that was viewed by the experimenter. The participant sat inside a small testing booth so that the experimenter and the participant had no direct visual contact with each other. In order to indicate eye movements, the experimenter had a box with two buttons, one for leftward eye movements and one for rightward eye movements. This button box was connected to the computer running the experiment, and buttonpresses were recorded concurrently with participants' responses. The experimenter was alerted to the critical point in the trial by a sound played through headphones, which came on briefly at the onset of the fixation cross, and again at the participant's response. If any movement of the eyes occurred during this interval, the experimenter indicated its direction by pressing the corresponding button.

The experimenters' accuracy in detecting eye movements was assessed via their performance during evaluation sessions, in which a participant made cued eye movements (or no eye movement) to a target at a particular location relative to fixation. Both experimenters were found to be essentially perfect in detecting all eye movements greater than $1.8^{\circ}$ of visual angle.

\section{Results and Discussion}

The participants made eye movements on $3.2 \%$ of the trials, and the data from these trials were eliminated from all further analyses. The results of the experiment, including only trials in which no eye movements were made, are graphed in Figure 7. An ANOVA was performed on the factors of orientation, figural status, and SOA. The results exactly mirrored those from Experiment 1 (see Figure 2), clearly demonstrating that the figural advantage in speed of detection does not depend on eye movements. There was a large main effect of figure-ground condition, such that participants were faster at detecting the target on the figural side than on the ground side $[F(1,6)=$ $38.83, p<.001]$. There was a main effect of SOA, such that participants were faster at longer SOAs $[F(3,18)=$ $28.79, p<.001]$. There was no interaction between orientation and figure $[F(1,6)<1]$ or between SOA and figure $[F(3,18)=1.61, p>.05]$.

\section{GENERAL DISCUSSION}

The results of the five experiments presented here are consistent with the hypothesis that figural cues in figureground displays draw attention to the side that is biased to be perceived as figure. This is strong evidence of a close relation between attention and figure-ground organization, in that the same cue of meaningfulness that strongly influences figural status also influences the allocation of attention along a contour in just the way one would expect if attention is drawn by figural cues to the figural region. We cannot tell from the present results whether the influence of this figural cue on attention is direct or whether it is mediated by the assignment of figural status per se. We can tell that this allocation of attention appears to be essentially automatic, however, because in all of these experiments, the target was equally likely to be located in the figure or in the ground, and thus provided no task-related advantage for attending to the figure.

We expected the figural advantage to be diminished when the target was presented on inverted familiar shapes in comparison with upright ones, but this effect occurred 
reliably only in Experiment 2, when participants explicitly reported which side they perceived as figure on some trials. We note that reductions in the likelihood that inverted familiar shapes are perceived as figure have been reported previously in explicit figure-ground tasks (e.g., Peterson \& Gibson, 1994; Peterson et al., 1991). It may be that such inversion effects are more likely to occur when an explicit response to the figural side is required than when it is not, but the present experiments cannot resolve this issue with certainty, because they do not manipulate this factor in isolation. We also note that inversion effects are most likely to be obtained when participants see the same object only once and when the displays are presented only briefly and masked immediately. Given that our participants viewed the objects for an extended time on each trial and did so many times during the course of the experiment, it is perhaps less surprising that we did not find robust inversion effects.

Somewhat surprisingly, the attentional effects we found operated only near the dividing contour. In particular, Experiment 3 showed that only targets within a short distance of the contour produced a figural RT advantage in detection. This finding suggests that figure-ground organization may be better understood as influencing perceptual processing along the contour than as doing so throughout the entire region attached to that contour. For this reason, it would be inaccurate to summarize the present results as indicating that people attend more to "the figure," as though the effect were uniformly present for the entire region. At least to the extent that detection time and discrimination accuracy are valid indices of attentional deployment, our data imply that attention is concentrated along the inner edge of the figural side of the shared contour.

The attentional advantages we found are broadly consistent with ecological considerations. Given that figural cues are correlated both with the closer side and with the side that is physically shaped by the shared contour, it makes sense that attention should be drawn to this side. All else being equal, closer objects are of more immediate evolutionary concern to a sighted organism than farther objects, because they are likely to be encountered sooner and are correspondingly more important in planning locomotion, haptic exploration, and other motor activity. Given that attentional shifts precede and may well drive overt eye movements (e.g., Hoffman \& Subramaniam, 1995; Rizzolatti, Riggio, Dascola, \& Umiltà, 1987), it is also reasonable to suppose that there would be evolutionary advantages to fixating closer objects than more distant background surfaces.

It is also true that perceptual predictions based on the shape of the shared contour are stronger and have greater validity for the properties of the closer surface (corresponding to the figural region) than for those of the farther surface (corresponding to the ground region). For example, if the shared contour is an extremal edge of an object that smoothly occludes itself along its curved surface, certain patterns of shading, highlights, and texture are likely to be present on the closer side and not on the farther side (Ghose \& Palmer, 2005). It is also true that the contour is more likely to share properties with texture on the figural side (e.g., motion and blur) than on the ground side, and that these similarities are likely to decrease with distance from the contour (Palmer \& Brooks, 2004). Note that the latter ecological considerations provide plausible rationales for the distance effect that was obtained in Experiment 3: The attentional advantage was stronger close to the shared contour and disappeared toward the interior of the figural region. The results we have found are thus broadly consistent with what might be expected from ecological considerations of the evolutionary benefits that might accrue to an organism that is biased to attend to the closer, shaped side (i.e., the figural region) rather than the farther, unshaped side (i.e., the ground region).

Quite independently, Lazareva, Castro, Vecera, and Wasserman (2006) recently reported a related finding in pigeons. When pigeons must report the location of a target spot as being either on the figure or on the ground, by a discriminative pecking response, they learned the task more quickly and performed it more rapidly when the spot was on the figure than when it was on the ground. The figural advantage disappeared, however, if the pigeons simply had to respond as soon as they saw the spot, without having to report where it was located. The latter result shows that pigeons do not exhibit the same figural advantage that we predicted (and found) for human performance, because the location of the target is entirely irrelevant in our tasks.

In a more limited theoretical framework, but one that can be tied explicitly to the results of the present experiments, our findings can be related to the existing hypothesis by Yantis and colleagues (Yantis \& Hillstrom, 1994; Yantis \& Jonides, 1984) that attention is drawn automatically to the sudden onset of a new perceptual object. These researchers did not consider figural cues in their definition of what constitutes "a new perceptual object," but such cues are clearly relevant to this issue. The process of determining whether a contour belongs to one region or another is about assigning it to the object (the figural side) rather

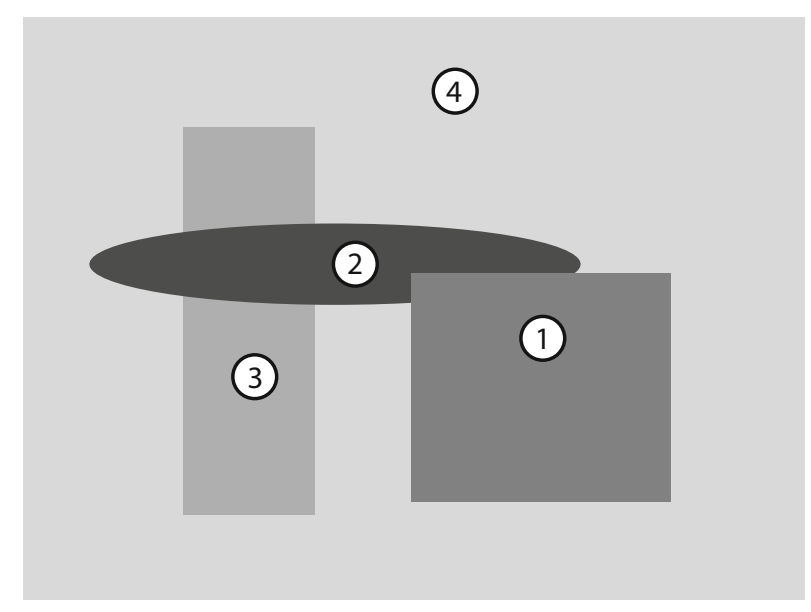

Figure 8. Figural status is always relative to a specific contour, as illustrated in this more complex display. Region 1 is always figure and Region 4 is always ground, whereas Regions 2 and 3 are figure or ground with respect to different parts of their contours. 
than to the adjacent space next to the object (the ground side; see Palmer, 1999, pp. 283-284). Since one region owns the contour, whereas the other region continues behind it, only one region is "the object," with respect to the contour. Thus, when a bipartite figure-ground display, such as those used in the present experiments, suddenly appears, only one new object actually appears - namely, the figure. ${ }^{3}$ The ground is not likely to be perceived as the appearance of another object, but rather as an unstructured space or surface that continues amodally behind the single perceived object. Presumably, an object-based form of attention would be responsible for this effect rather than a purely spatial form of attention, because the results of figure-ground organization concern the shapes of regions perceived as objects. ${ }^{4}$

It is important to understand that it is only locally with respect to a contour that a region is figure or ground. From this perspective, Rubin's original discussions of figureground perception, as well as the way it is discussed in many textbooks, are somewhat misleading. The real world does not generally consist of complete figures against meaningless, shapeless grounds. Rather, it is often the case that there are many objects that occlude each other in complex ways. Figure 8 shows an example in which there are more objects in different depth planes than are seen in traditional bipartite displays. It makes more sense to determine the figural status of the local region with respect to a contour. For example, with respect to the contour bordering Region 1 and Region 2, Region 1 is the figure. However, with respect to the contour bordering Region 2 and Region 3, Region 2 is the figure. We do not claim that figure-ground relations are only about bordering contours, however. The results of figure-ground organization are relevant to the perceptual interpretation of two-dimensional regions in an image, rather than the one-dimensional borders around them, but it only makes sense to define a region as figural with respect to a given contour, and the present results suggest that this fact is reflected in the restriction of the figural advantage to areas near the contour. ${ }^{5}$

The results of the present experiments warrant the conclusion that attention should be treated as an important aspect of the figure-ground phenomenon, along with depth, shape, and amodal completion. Not only do figural cues bias which side will be seen as closer and shaped, versus farther and completed, but they appear to automatically allocate attention to the area near the border on the figural side. Even so, there are several important issues that need to be resolved by further research. One is whether the attentional advantage we found in all four experiments with meaningful shapes will also be present for the classical "bottom-up" cues to figure-ground organization, such as surroundedness, size, symmetry, convexity, and lower region. We are currently examining this question in parallel experiments using novel, meaningless contours.

Another important unresolved issue is whether the figural advantage we found is due to the direct influence of figural cues on attention or whether it is mediated by figure-ground organization. Although these two possibilities are difficult to tease apart, it may be possible to do so by asking participants to attend to the ground region on some trials and to the figural region on others. If the processing advantages we measured are due purely to figural status, then it should not matter whether participants are attending to the figure or the ground side: The advantages should always accrue to the figural side and remain there. If it is due to attention, then the advantages noted here should eventually accrue to the ground side on trials in which participants are instructed to attend to the ground, but not on trials in which they are instructed to attend to the figure. We are also engaged in the process of answering this question.

\section{AUTHOR NOTE}

This research was supported, in part, by NIMH Grant 1-R01-MH46141 and by a grant from the Swanton Foundation. The authors thank Joseph Brooks and William Prinzmetal for comments on the manuscript. Correspondence concerning this article should be addressed to R. A. Nelson, Wheaton College, Norton, MA 02766 (e-mail: rnelson@wheatonma .edu).

\section{REFERENCES}

BAYlis, G. C., \& Driver, J. (1995). One-sided edge assignment in vision: I. Figure-ground segmentation and attention to objects. Current Directions in Psychological Science, 4, 140-146.

Christie, J., \& Klein, R. (1995). Familiarity and attention: Does what we know affect what we notice? Memory \& Cognition, 23, 547-550.

Coren, S., WARD, L. M., \& EnNs, J. T. (1999). Sensation and perception (5th ed.). Fort Worth, TX: Harcourt Brace.

CRAIK, K. J. W., \& ZANGWILL, O. L. (1939). Observations relating to the threshold of a small figure within the contour of a closed-line figure. British Journal of Psychology, 30, 139-150.

Davis, J. M. (1985). An examination of the status of the ground in figure-ground perception. Unpublished doctoral dissertation, Rutgers University.

Gelb, A., \& Granit, R. (1923). Die Bedeutung von "Figur" und "Grund" für die Farbenschwelle. Zeitschrift für Psychologie, 93, 83-118.

Ghose, T., \& Palmer, S. E. (2005, May). Surface curvature and extremal edges in depth perception and figure-ground organization. Paper presented at the 5th Annual Meeting of the Vision Science Society, Sarasota, FL.

Green, D. M., \& Swets, J. A. (1966). Signal detection theory and psychophysics. New York: Wiley.

Hansen, C. H., \& Hansen, R. D. (1988). Finding the face in the crowd: An anger superiority effect. Journal of Personality \& Social Psychology, 54, 917-924.

Hoffman, J. E., \& Subramaniam, B. (1995). The role of visual attention in saccadic eye movements. Perception \& Psychophysics, 57, 787-795.

Hupé, J. M., James, A. C., Payne, B. R., Lomber, S. G., Girard, P., \& Bullier, J. (1998). Cortical feedback improves discrimination between figure and background by V1, V2, and V3 neurons. Nature, 394, 784-787.

JonidES, J. (1980). Towards a model of the mind's eye's movement. Canadian Journal of Psychology, 34, 103-112.

JONIDES, J. (1981). Voluntary versus automatic control over the mind's eye. In J. [B.] Long \& A. [D.] Baddeley (Eds.), Attention and performance IX (pp. 187-203). Hillsdale, NJ: Erlbaum.

JoNIDES, J. (1983). Further toward a model of the mind's eye's movement. Bulletin of the Psychonomic Society, 21, 247-250.

KoffKa, K. (1935). Principles of Gestalt psychology. New York: Harcourt, Brace.

LAMme, V. A. F. (1995). The neurophysiology of figure-ground segregation in primary visual cortex. Journal of Neuroscience, 15, 1605-1615.

Lazareva, O. F., Castro, L., Vecera, S. P., \& Wasserman, E. A. (2006). Figure-ground assignment in pigeons: Evidence for a figural benefit. Perception \& Psychophysics, 68, 711-724.

LEVIN, D. T. (1996). Classifying faces by race: The structure of face categories. Journal of Experimental Psychology: Learning, Memory, \& Cognition, 22, 1364-1382. 
Matuin, M. W. (1983). Perception. Boston: Allyn \& Bacon.

Maurer, D., Le Grand, R., \& Mondloch, C. J. (2002). The many faces of configural processing. Trends in Cognitive Sciences, 6, 255-260.

OsGood, C. E. (1953). Method and theory in experimental psychology. New York: Oxford University Press.

Palmer, S. E. (1999). Vision science: Photons to phenomenology. Cambridge, MA: MIT Press.

Palmer, S. E., \& Brooks, J. L. (2004, May). Edge-texture grouping: A new cue to edge assignment. Paper presented at the 4th Annual Meeting of the Vision Science Society, Sarasota, FL.

Palmer, S. [E.], \& Rock, I. (1994). Rethinking perceptual organization: The role of uniform connectedness. Psychonomic Bulletin \& Review, 1, 29-55.

Peterson, M. A. (1999). What's in a stage name? Comment on Vecera and O'Reilly (1998). Journal of Experimental Psychology: Human Perception \& Performance, 25, 276-286.

Peterson, M. A., de Gelder, B., Rapcsak, S. Z., Gerhardstein, P. C., \& Bachoud-LÉvi, A.- C. (2000). Object memory effects on figure assignment: Conscious object recognition is not necessary or sufficient. Vision Research, 40, 1549-1567.

Peterson, M. A., Gerhardstein, P. C., Mennemeier, M., \& Rapcsak, S. Z. (1998). Object-centered attentional biases and object recognition contributions to scene segmentation in left- and right-hemisphericdamaged patients. Psychobiology, 26, 357-370.

Peterson, M. A., \& Gibson, B. S. (1993). Shape recognition inputs to figure-ground organization in three-dimensional displays. Cognitive Psychology, 25, 383-429.

Peterson, M. A., \& Gibson, B. S. (1994). Must figure-ground organization precede object recognition? An assumption in peril. Psychological Science, 5, 253-259.

Peterson, M. A., Harvey, E. M., \& Weidenbacher, H. J. (1991). Shape recognition contributions to figure-ground reversal: Which route counts? Journal of Experimental Psychology: Human Perception \& Performance, 17, 1075-1089.

Posner, M. I. (1978). Chronometric explorations of mind. Hillsdale, NJ: Erlbaum.

PosNer, M. I. (1980). Orienting of attention. Quarterly Journal of Experimental Psychology, 32, 3-25.

Posner, M. I., Nissen, M. J., \& Ogden, W. C. (1978). Attended and unattended processing modes: The role of set for spatial location. In H. L. Pick \& E. Saltzman (Eds.), Modes of perceiving and processing information (pp. 137-157). Hillsdale, NJ: Erlbaum.

Prinzmetal, W., Nwachuku, I., Bodanski, L., Blumenfeld, L., \& Shimizu, N. (1997). The phenomenology of attention: 2. Brightness and contrast. Consciousness \& Cognition, 6, 372-412.

Rizzolatti, G., Riggio, L., Dascola, I., \& Umiltà, C. (1987). Reorienting attention across the horizontal and vertical meridians: Evidence in favor of a premotor theory of attention. Neuropsychologia, 25, 31-40.

Rubin, E. (1958). Figure and ground. In D. Beardslee \& M. Wertheimer (Eds. \& Trans.), Readings in perception (pp. 35-101). Princeton, NJ: Van Nostrand. (Original work published 1915)

Sternberg, R. J. (1996). Cognitive Psychology. Fort Worth, TX: Harcourt Brace.
Sugihara, T., Qiu, F. T., \& von DER Heydt, R. (2004). Figure-ground organization and attention modulation in neurons of monkey area $\mathrm{V} 2$ [Abstract]. Journal of Vision, 4, 197a.

Vecera, S. P., Flevaris, A. V., \& Filapek, J. C. (2004). Exogenous spatial attention influences figure-ground assignment. Psychological Science, 15, 20-26.

Vecera, S. P., \& O'Reilly, R. C. (1998). Figure-ground organization and object recognition processes: An interactive account. Journal of Experimental Psychology: Human Perception \& Performance, 24, 441-462.

VON DER Heydt, R., Zhou, H., \& Friedman, H. S. (2000). Representation of stereoscopic edges in monkey visual cortex. Vision Research, 40, 1955-1967.

Wong, E., \& WeIsSTEIN, N. (1982). A new perceptual context-superiority effect: Line segments are more visible against a figure than against a ground. Science, 218, 587-589.

Yantis, S., \& Hillstrom, A. P. (1994). Stimulus-driven attentional capture: Evidence from equiluminant visual objects. Journal of Experimental Psychology: Human Perception \& Performance, 20, 95-107.

Yantis, S., \& Jonides, J. (1984). Abrupt visual onsets and selective attention: Evidence from visual search. Journal of Experimental Psychology: Human Perception \& Performance, 10, 601-621.

\section{NOTES}

1. The reader is referred to Prinzmetal, Nwachuku, Bodanski, Blumenfeld, and Shimizu (1997) for a review of this claim and for experimental evidence indicating that attention does not, in fact, influence brightness or contrast perception.

2. This is different from the claim that intent influences what is figure. Rubin (1915/1958) noted that an otherwise ambiguous region might be perceived as a figure merely by willing it. However, this is different than spatial attention to a region. It is perfectly consistent to attend to a region while intending to perceive it as ground

3. In an important sense, then, the allocation of attention is different than that found in experiments in which one meaningful object is given attentional priority over other objects distributed in a scene (Christie \& Klein, 1995; Hansen \& Hansen, 1988; Levin, 1996).

4. Palmer and Rock (1994), in their theoretical framework of perceptual organization, interpreted the output of figure-ground processes to be entry-level units in a region map of the image. These units are partitions of uniformly connected regions of a visual image, segregated according to figure-ground principles. With respect to their contours, each region would presumably have the aspects of figure-ground discussed above.

5. The basis of figure-ground cues and the result of figure-ground organization should not be confused with each other. Although the cues influencing figural assignment (see below) may be based primarily on properties of the region (e.g., surroundedness or size) or properties of the contour (e.g., meaningfulness or convexity), the resulting percept is always that of a figural region with respect to a given contour.

(Manuscript received June 30, 2005; revision accepted for publication August 7, 2006.) 